\title{
Physical Activity, Exercise and Breast Cancer - What Is the Evidence for Rehabilitation, Aftercare, and Survival? A Review
}

\author{
Petra Wirtz $^{\mathrm{a}, \mathrm{b}} \quad$ Freerk T. Baumann ${ }^{\mathrm{a}}$ \\ a Department I of Internal Medicine, Center of Integrated Oncology Cologne Bonn, University Hospital of Cologne, Cologne, Germany; \\ ${ }^{b}$ German Sport University Cologne, Cologne, Germany
}

\section{Keywords}

Physical activity · Breast cancer · Rehabilitation · Aftercare

\section{Summary}

The current scientific field of exercise interventions in breast cancer is very large. Rehabilitation focuses on restoring or improving quality of life and thus on independence and participation in life. Hence, it is important to investigate exercise interventions in the setting of rehabilitation and aftercare. It is also necessary to determine the influence of physical activity or exercise therapy on the prevention of recurrence and mortality as well as on certain side effects of the disease and its medical treatment in rehabilitation. Physical activity in rehabilitation and aftercare is feasible and safe and can be used for various purposes. Against the background of reduced fatigue, chemotherapy-induced peripheral neuropathy, cancer-related cognitive impairment, arthralgia, lymphedema, recurrence, and mortality, as well as improved quality of life, different methodological approaches and evidence can be seen. Although the overall data availability has increased, there are gaps in study documentation as well as inadequate verification of scientific approaches in rehabilitation clinics and in everyday life.

(C) 2018 S. Karger GmbH, Freiburg

\section{Introduction}

According to the GEKID (Network of Cancer Registries in Germany) Atlas, the incidence rate for breast cancer in Germany has decreased [1]; however, the rate is still high and women are confronted with cancer- and treatment-related side effects $(67,420$ women in 2014). The increasing number of worldwide studies on physical activity and breast cancer highlights the importance given to this correlation.

Patients with breast cancer can receive various forms of medical therapy such as surgery, chemotherapy, hormone therapy, antibodies, and/or radiotherapy and be confronted with various side effects such as fatigue [2]. In addition, breast cancer patients start to decrease their physical activity during therapy [3] and stay physically inactive during aftercare [4]. However, physical activity is a possible adjuvant therapy for cancer $[5,6]$.

Physical, psychological, and social impairments can be reduced with aerobic and resistance training [7], and regular exercise has an influence on acute and chronic symptoms of breast cancer [8]. Especially specifically structured aerobic and resistance exercises have a positive influence [5]. Physical activity can have a positive influence on insulin sensitivity, immune and cardiopulmonary function, bone loss, body composition, depression, sleep disturbances, and quality of life in the aftercare of breast cancer $[8,9]$. Long-term physical effects are associated with and are depending on the type of medical treatment for breast cancer patients after oncological therapy $[8,10]$.

The key aim of rehabilitation is to make the patient fit for everyday life. An individual focus is crucial for this. Particularly in the rehabilitation and aftercare of breast cancer survivors, it is necessary to prepare for everyday life and lay the foundation for a physically active lifestyle that also considers prevention of recurrence, increased risk of comorbidities, and aspects of survival [4]. Above

\section{KARGER}

() 2018 S. Karger GmbH, Freiburg
PD Dr. Freerk T. Baumann 


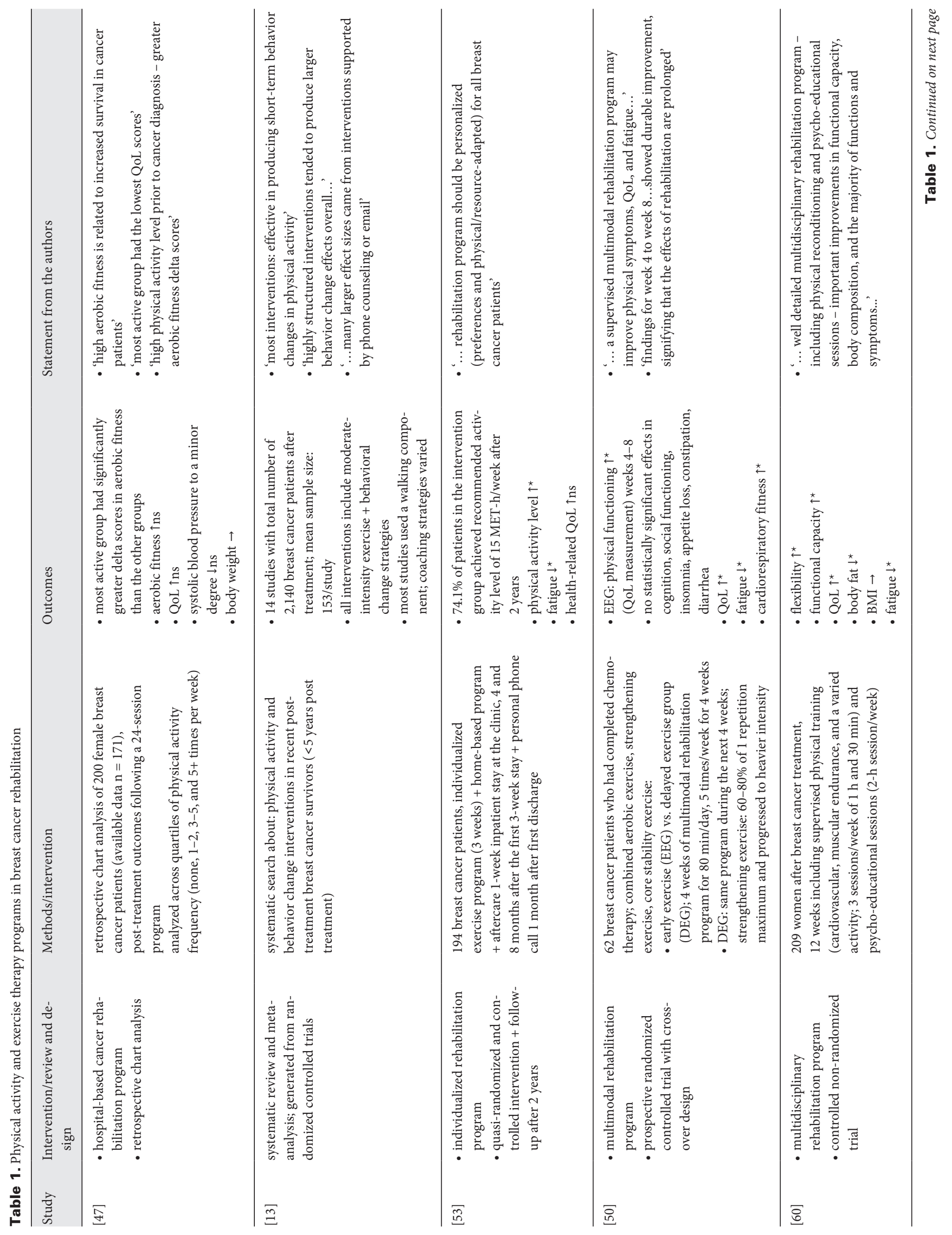




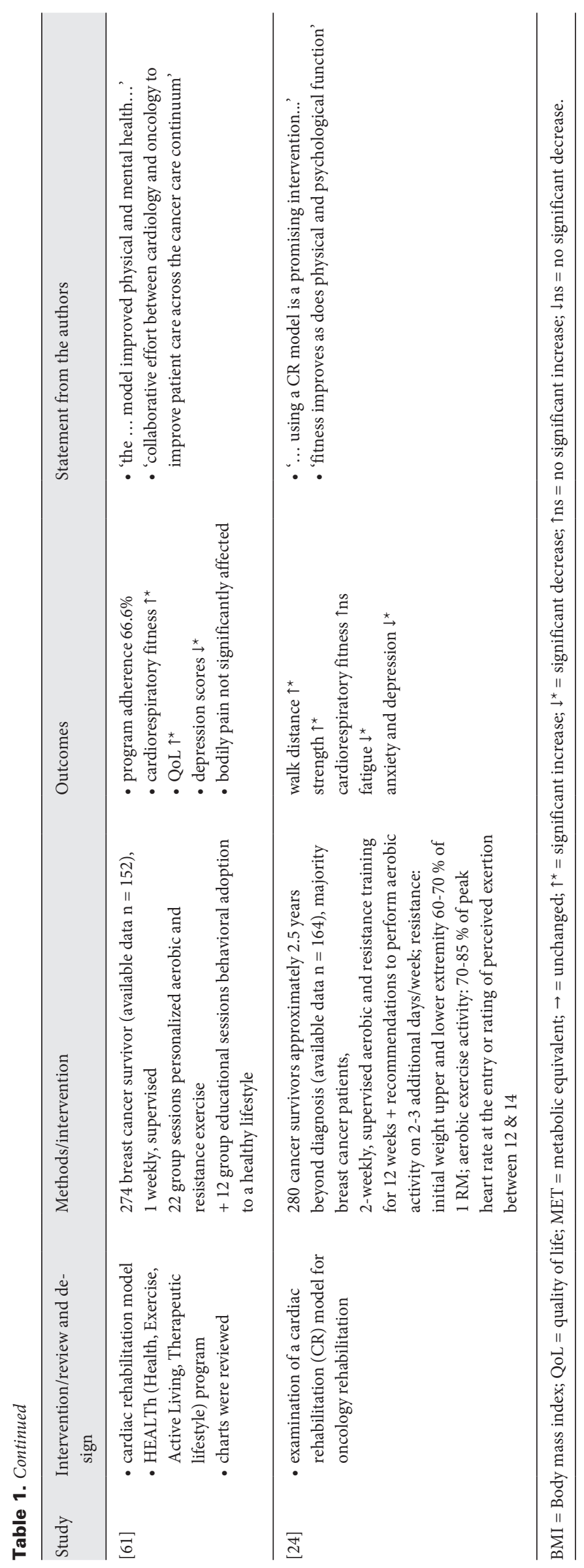

all, the restoration or improvement of quality of life should be the focus of the intervention's design and should be meaningful. Quality of life is influenced by different individual aspects as well as by cancer- and treatment-related side effects. Additionally, studies on exercise in women with breast cancer are often incomplete and not clear with regard to the methods reported [11].

Therefore, the aim of this article was to review the current data from which exercise recommendations are generated and discuss targeted exercise programs and recommendations for rehabilitation and aftercare in breast cancer survivors, focusing on certain side effects, quality of life, and impact on recurrence and survival. On the one hand, side effects such as fatigue, chemotherapy-induced peripheral neuropathy (CIPN), cancer-related cognitive impairment (CRCI), arthralgia, and lymphedema are considered since these influence the direction of relevant exercise interventions during the rehabilitation phase in breast cancer patients. There are approaches that investigate the already determined influences of physical activity on cancer cachexia [12]. However, as this plays a minor role in exercise therapy in the rehabilitation and aftercare phase of breast cancer, it is not discussed in detail. On the other hand, the crucial factors of recurrence and survival are considered in relation to aftercare.

To investigate the current study situation, the platforms NCBI PubMed and Google Scholar were used and the literature of the most recent systematic reviews analyzed. As a qualitative analysis, the results partially contain direct quotes or statements from the authors.

\section{Physical Activity Interventions and Programs during Rehabilitation and Aftercare}

There are few studies investigating programs or physical activity interventions during the rehabilitation phase. These show different options for patients in rehabilitation and aftercare, but also different study designs and reported information (table 1). Particularly for programs conducted during rehabilitation, statements and evidence vary widely. A systematic review with 14 randomized controlled trials (RCTs) demonstrated good evidence for short-term effects [13] but also illustrated partially large differences between interventions, which can also be found in the table shown here (table 1). Another systematic review with 10 studies described the initial evidence of the feasibility and efficacy of exercise in terms of improvement of physiological and psychological function during the rehabilitation phase [14].

Next to supervised physical activity interventions, other studies with small sample sizes examined different ways for cancer patients to participate in health-related programs. For example, Cox et al. [15] compared in an RCT a 6-month internet- and telephone-delivered weight loss intervention with 37 cancer survivors. The health outcomes of the cancer survivors were better in the telephone group. Ritvo et al. [16] are going to evaluate a 12-week physical activity intervention with 107 physically inactive breast cancer survivors ( $<60 \mathrm{~min} /$ week), also in an RCT design, to inform full- 
Table 2. Recommendations/guidelines for breast cancer survivors from different reviews $[10,55,62,63]$
- Strength training exercises 2 times/week

- $150 \mathrm{~min}$ of moderate or $75 \mathrm{~min}$ of vigorous physical activity/week

- Normal daily activities as soon as possible

- Prevent inactive lifestyle

- Advise patients about healthy weight

- Emphasize strength training for women treated with adjuvant chemotherapy or hormone therapy

- Need for nutrition counseling

- Need for physical activity

- Need for weight management

scale RCT planning. Next to weekly group sessions, participants will receive a home-based program, telephone health coaching, smartphones with data plans, supportive health tracking software, and wearables. Also, a systematic review with 14 randomized studies, and most of the included studies with breast cancer survivors, reports that group or community-based activities with approaches to behavioral change have the biggest effect. Home- and community-based physical activity programs could improve functional deterioration in aging cancer survivors [17].

Currently available studies also provide initial information on the timing of exercise interventions and associated effects in aftercare. For example, a nutrition and exercise intervention (supervised moderate intensity, aerobic and resistance training, 3 times/ week) during adjuvant chemotherapy in 73 breast cancer patients showed long-term effects of improved physical activity levels and quality of life 2 years after the start of therapy [18].

The length of an intervention as well as its intensity are crucial factors in program planning. Schmitt et al. [19] showed that lowto-moderate intensity as well as high-intensity interval training can have the same positive influence on fatigue, quality of life, and physical factors in female cancer survivors. The high-intensity interval training was therefore feasible and could be used with lowtime approaches in cancer survivors.

One study could even show that a guided adjuvant high-intensity interval endurance and strength training could be feasible and shows an improvement in endurance and strength performance and quality of life in breast cancer patients after only 6 weeks [20].

Studies with low numbers of subjects repeatedly show feasibility and various improvements but lack good evidence (e.g., ashtanga yoga [21] and dragon boat activity [22]).

Also, approaches taken from other diseases were examined for cancer survivors, and studies have demonstrated usability of existing cardiac rehabilitation structures also in cancer patients. One single-arm study indicated that an oncology rehabilitation exercise program with 20 breast cancer patients could be integrated in a cardiac rehabilitation program, but did not meet the primary outcome in terms of adherence and significant changes in aerobic exercise capacity [23]. Dittus et al. [24] showed that topics like exercise training, physical activity, nutrition counseling, psychosocial management, and weight management are the same in cancer and cardiac rehabilitation, also demonstrating usability of cardiac rehabilitation structures.

Despite the different approaches and outcomes of the studies shown, several recent reviews provide general recommendations independent of specific side effects (table 2).

\section{Quality of Life}

Especially in rehabilitation and aftercare, the focus is on the reduction and improvement of cancer- and treatment-related side effects that do not subside even after the end of the therapy. Depression and fatigue are associated with impaired quality of life, and quality of life is impaired not long after adjuvant treatment in breast cancer patients [25]. Mustian et al. [26] showed in an overview of the oncology literature on the use of exercise that a lot of programs aim to restore and improve the quality of life of cancer survivors.

A survey with 537 breast cancer survivors demonstrated physical performance and activity level having a positive correlation with quality of life [25]. Duncan et al. [27] presented in a review of 21 systematic reviews of randomized trials that physical exercise interventions (yoga, aerobic and resistance training) are effective in improving overall quality of life in cancer survivors. There seems to be no standard program, but supervised interventions and a combination of aerobic and resistance training appear more effective (table 1). Organized specific programs could improve quality of life in breast cancer survivors in a specific exercise intervention with 55 women [28].

\section{Fatigue}

Mustian et al. [29] expounded that exercise therapy is effective in reducing cancer-related fatigue during and after cancer treatment. Exercise therapy and psycho-oncology are significantly more effective than pharmaceutical interventions. They examined 113 randomized clinical studies with 11,525 patients. Zimmer et al. [30] with a rehabilitation program including 60 patients and MenesesEchavez et al. [31] with a systematic review of 14 RCTs showed that physical activity levels can increase with personalized exercise recommendations and supervised exercise interventions among breast cancer patients. The recommended training approaches and intensities are based on the level of fatigue (mild, moderate, or severe) in cancer survivors. Generally, a combination of aerobic and resistance exercise may be recommended according to an overview [32]. According to a systematic review with 25 RCTs, there was slightly better improvement in fatigue when breast cancer patients took part in physical exercise intervention after adjuvant treatment [33].

\section{Chemotherapy-Induced Peripheral Neuropathy}

Streckmann et al. [34] showed in a systematic review with 18 studies (10 RCTs and 8 controlled clinical trials) that balanced training is more effective than a combination of endurance and strength training for patients with peripheral neuropathies. Overall, exercise can reduce symptoms of CIPN especially in breast cancer patients. In line with this, a multicenter RCT with a 6-week standardized and home-based progressive walking and resistance exercise intervention with moderate intensity could improve CIPN symptoms in 355 cancer patients receiving taxane-, platinum-, or vinca alkaloid-based chemotherapy [35]. 


\section{Cancer-Related Cognitive Impairment}

A systematic review with 19 studies (rodents and humans) presented that physical activity such Asian-influenced movement programs could have a positive influence on self-perceived cognitive functions in patients with breast cancer in aftercare; it may also reduce chronic inflammation [36]. The review suggests that exercise interventions can help in the prevention and rehabilitation of CRCI and improve processing speed with early implementation (diagnosis $<2$ years) among breast cancer patients [37]. However, the evidence and quality of the studies in humans are poor.

\section{Secondary Lymphedema}

Interventional studies show that physical activity is not contraindicated in patients with breast cancer-related lymphedema and that subjective and objective parameters can be positively influenced by high-frequency dynamic and moderate training [38]. For example, pain and loss of sensitivity can be improved by strength training [39]. Water and home-based training could improve functional limitations in the affected arm as well as quality of life [40].

\section{Arthralgia}

The specific effects and mechanisms of certain exercise programs on arthralgia have not yet been sufficiently investigated. Physical activity can have a positive influence on aromatase inhibitor-associated arthralgia. For example, after 1 year, endurance and strength training had a positive effect on pain intensity and pain intervals in a trial with breast cancer patients [41]. However, further investigations are needed to determine the nature of the effect and above all which methods and intensities should be applied [42].

\section{Cardiotoxicity}

A prospective cohort study with non-metastatic breast cancer patients showed that exercise is associated with a reduction in the incidence of cardiovascular events [43]. Runowicz et al. [10] recommend among other things early cardiovascular monitoring and education of breast cancer survivors about lifestyle adaptations including exercise. This could have a positive influence on the risk or severity of cardiotoxicity or cardiovascular events.

\section{Physical Activity Interventions and Programs Related to Recurrence and Survival}

How exactly physical activity pre- or post-diagnosis contributes to reducing recurrence and/or mortality is still not fully understood [5]. A number of studies have examined certain criteria and come to certain conclusions (table 3). However, different approaches in methodology, scope, and outcome have yielded heterogeneous evidence.

Emaus et al. [44] determined a relationship between mortality and body mass index, blood pressure, lipids, and physical activity in a population-based study including 1,364 breast cancer cases. A prospective observational study and large RCTs proved that not only fulfilling the guidelines of physical activity but also the timing (pre- or post-diagnosis/treatment) and duration of an active lifestyle affect the survival and risk of recurrence in women with breast cancer $[45,46]$. It was demonstrated that a physically inactive lifestyle (very low physical activity) is associated with a higher risk of mortality than obesity post-diagnosis. Adamo et al. [47] showed in a hospital-based cancer rehabilitation program with 171 women that a high degree of aerobic fitness was related to improved survival.

There are also isolated studies investigating the mechanical effects of physical activity at the cellular and molecular level, but sample sizes are small and the evidence is poorly understood. The endogenous immune system could play a central role in the defense against malignant cells [48]. Physical exercise could have a protective influence in terms of recurrence prophylaxis. After a 6-month exercise training with 28 breast cancer patients in aftercare, increased lymphocyte activation could be detected [49].

\section{Discussion and Conclusion}

The aim of this review was to provide an overview of the current publications, especially their evidence, about physical activity in rehabilitation and aftercare in breast cancer patients. Various topics and different outcome reports were encountered.

There are many studies with various aims and recommendations for physical activity in breast cancer survivors. It appears that exercise can positively influence certain cancer- and treatment-related side effects, quality of life, recurrence, and survival. However, there are differences in outcomes depending on factors such as intensity and activity level. A high level of aerobic fitness can be associated with a higher probability of survival; however, a high level of activity is not necessarily associated with the best quality of life [47].

There is differentiated evidence with regard to exercise against certain side effects.

The positive effect of exercise on fatigue has been verified in RCTs and systematic reviews with strong evidence. It is now a matter of integrating or implementing the approaches into the daily practice of rehabilitation clinics.

As far as CIPN is concerned, evidence-based approaches with RCTs and reviews show that exercise has a positive effect on symptoms, but there is still a lack of larger RCTs and programs closer to daily life.

The validity of physical activity against cognitive impairment among breast cancer patients is not well understood and there is no reliable evidence for certain movement recommendations. More RCTs with human cancer patients are required.

Many exercise programs and interventions are aimed at improving quality of life, and there is now good study-based evidence. In this context, methodology still differs considerably. Ultimately, the efficacy of scientific approaches must also be checked in practice, and here evidence is still too low. 
Table 3. Studies about the influence of physical activity (PA) on recurrence and survival

\begin{tabular}{|c|c|c|}
\hline Study & Design & Recommendations/statement from the authors \\
\hline [64] & $\begin{array}{l}\text { - meta-analysis of published studies } \\
\text { - included } 6 \text { studies with } \\
12,108 \text { breast cancer patients }\end{array}$ & $\begin{array}{l}\text { - 'post-diagnosis PA reduced } \\
\text { - breast cancer deaths by } 34 \% \\
\text { - all causes mortality by } 41 \% \\
\text { - disease recurrence by } 24 \% \text { ' } \\
\text { - '... evidence for an inverse relationship between PA and mortality ...' }\end{array}$ \\
\hline [5] & - review of evidence & $\begin{array}{l}\text { - 'certain patient subtypes are more responsive to physical activity than others' - 'observe/note possible } \\
\text { responsiveness to exercise' } \\
\text { - 'strongest evidence for an association between post-diagnosis physical activity and cancer-specific } \\
\text { mortality is found for breast and colon cancers for which the average mortality risk decrease is } 25 \text { and } \\
32 \% \text {, respectively' }\end{array}$ \\
\hline [65] & $\begin{array}{l}\text { - meta-analysis of cohort studies } \\
\text { - included } 16 \text { cohort studies with } \\
42,602 \text { breast cancer patients }\end{array}$ & $\begin{array}{l}\cdot \text { '. . both pre-diagnosis and post-diagnosis PA were associated with reduced breast cancer-specific } \\
\text { mortality and all-cause mortality' }\end{array}$ \\
\hline [9] & - review & $\begin{array}{l}-\ldots 24-67 \% \text { reduction in the risk of total deaths and } 50-53 \% \text { reduction in the risk of breast cancer deaths } \\
\text { in women who are physically active after breast cancer diagnosis compared with sedentary women' }\end{array}$ \\
\hline$[46]$ & $\begin{array}{l}\text { - prospective observational study of } \\
933 \text { women with breast cancer }\end{array}$ & $\begin{array}{l}\text { - } \geq 9 \text { metabolic equivalents (METs)/week (e.g., } 2-3 \mathrm{~h} / \text { week of brisk walking) - before and especially after di- } \\
\text { agnosis and treatment } \\
\text { - 'women who increased physical activity after diagnosis had a } 45 \% \text { lower risk of death' } \\
\text { - 'moderate-intensity physical activity after a diagnosis of breast cancer may improve prognosis' }\end{array}$ \\
\hline
\end{tabular}

[45] - randomized controlled trial with 2,361 post-treatment breast cancer survivors (stage I-III)
- '... most active at baseline had a 53\% lower mortality risk compared to the least active women. Adherence to activity guidelines was associated with a $35 \%$ lower mortality risk'

- ' $42 \%$ reduction in risk mortality among those women who were adhering to physical activity guidelines at both baseline and 1-year relative to those who did not meet the guideline at either time point'

- 'we did not find that 1-year change in physical activity was associated with breast cancer mortality'
[66] - systematic review and metaanalysis

- used PRISMA guidelines

- included 16 studies with breast cancer survivors and 7 studies with colorectal cancer survivors (49,095 cancer survivors)
- '.. engaging in the approximate equivalent of 150 min of at least moderate physical activity per week after cancer diagnosis was associated with a $24 \%$ reduced risk of total mortality among breast cancer survivors'

- 'comparable amount of physical activity performed pre-diagnosis was related to a $13 \%$ reduced total mortality risk among breast cancer survivors'

- ' $\ldots$ benefit of physical activity was evident for both lean and overweight women, for pre- and postmenopausal women, and for ER-positive and -negative tumors'

[67] - data collection from breast cancer survivor cohorts in a after breast cancer pooling project with 9,513 patients

[68] - prospective cohort study with 3,393 non-metastatic breast cancer patients

- 'very low physical activity was associated with a $22 \%$ increased risk of breast cancer mortality' - very low physical activity: '.. defined as physical activity $<1.5 \mathrm{MET}-\mathrm{h} /$ week...' and ' $\ldots$ equivalent to $<30 \mathrm{~min}$ of moderate paced walking per week'

- 'risk for recurrence was about 50\% reduced in patients with estrogen/progesterone receptor-negative (ER-/PR-) tumors who had been physically active pre-diagnosis, e.g., cycled or exercised at least $1 \mathrm{~h} /$ day, compared to physically inactive women'

- 'among patients with ER-/PR- breast tumors, higher levels of exercise may even reduce their risk of recurrence'

- '...there was no mortality reduction with increasing PA levels."

[69] - international open-label randomized controlled trial with 521 breast cancer patients

- 'overall survival was better for patients who were active before and after the diagnosis. In contrast with previous studies, breast cancer survival and the risk of recurrence were not significantly associated with physical activity'

[70] - systematic review and metaanalysis of epidemiological studies

- 22 prospective cohort studies with 123,574 participants

- '... significant associations between lifetime and recent pre-diagnosis recreational physical activity and risk of all-cause death...'

- '.. recent pre-diagnosis recreational physical activity was also found to be associated with the risk of breast cancer-related death'

- 'post-diagnosis physical activity was found to significantly reduce the risk of both all-cause death and breast cancer-related death'

[71] - prospective observational study based on responses

- from 2,987 breast cancer patients

- 'physical activity after a breast cancer diagnosis may reduce the risk of death from this disease. The greatest benefit occurred in women who performed the equivalent of walking $3-5 \mathrm{~h}$ per week at an average pace, with little evidence of a correlation between increased benefit and greater energy expenditure' 
A lot of RCTs and systematic reviews with large sample sizes showed that pre- and post-diagnosis physical activity was associated with a protective influence on recurrence and survival. Statistical differences and probabilities differ in the individual percentages.

Overall, there is still no specific exercise intervention for preventing recurrence or breast cancer-related mortality. Studies and reviews distinguish between the prevention of total mortality and cancer mortality or investigate the positive influences on total survival or cancer survival. However, from the patient's point of view, this makes no difference. In rehabilitation centers, exercise programs are less designed to directly prevent a recurrence but rather to improve quality of life and reduce different side effects from breast cancer and related treatments [50]. Therapists should consider the different recommendations and focus on the individual aim of the patient. All in all, therapists, nurses, and doctors play an important role in motivating and encouraging patients to participate in rehabilitation programs during/after therapy and in aftercare to help prevent recurrence and mortality $[9,51]$. Therapists or instructors in group-based interventions can also create a supportive climate and foster positive experiences [52], and participation in a rehabilitation intervention can lastingly increase the level of physical activity even after the intervention [3].

General recommendations for cancer survivors without a specific target still apply, e.g., 15 metabolic equivalents (METs) per week or 150 min of moderate activity per week and a combination of strength and aerobic exercise [9] (table 2), but many patients fail to keep up with these recommendations. Physical activity guidelines for cancer survivors should consider patients' interests and individual preferences to create more personalized physical activity programs $[6,53,54]$. Cancer survivors need a larger range of varied programs and access to different alternatives [55].

Stubblefield et al. [56] noted that there is incomplete knowledge about cancer rehabilitation in patients and referring clinicians. It is also interesting that treatment rates for remediable physical impairments in cancer rehabilitation are lower than $2 \%$ [57]. There are still too few cost-benefit analyzes for the implementation and conversion of rehabilitation programs [58]. However, physical activity can and should be integrated into regular routine care and should be adapted to individual circumstances among breast cancer survivors [59].

The current evidence on physical activity during rehabilitation and aftercare is good but not reflected in the rehabilitation clinics. Common rehabilitation guidelines in Germany (over 3 weeks) should be adjusted in terms of intervention and care as well as length of support [53]. Institutions in charge of rehabilitation should also support the evaluation of innovative approaches to make use of good evidence.

For the preparation and presentation of this review, we did not apply the methodology of a systematic review with subsequent limitations. No systematically traceable search criteria are represented, and no PRISMA criteria were considered. Furthermore, only 1 author conducted the search so that not all relevant studies may have been considered. Various findings included a wide range of evidence on different side effects as well as on recurrence and survival in breast cancer patients.

Despite the increasing number of publications, data show incomplete reporting and limitations in methodology. Overall, studies should focus on the implementation and reviewing of scientific evidence in daily practice [55]. Interventions must take into account the circumstances and settings of rehabilitation and aftercare.

To improve the evidence that physical activity can be used specifically in the rehabilitation and aftercare of breast cancer, more randomized and controlled studies are needed, especially about recurrence and survival. For a more detailed examination, underlying biological mechanisms should also be considered/analyzed.

Overall, personalized programs that can be used in practice and have the power to motivate patients to change or lastingly improve their lifestyle should be the future approach in breast cancer rehabilitation and aftercare. In addition, more studies should be carried out in rehabilitation clinics as the setting and evidence of physical activity are most relevant here.

\section{Disclosure Statement}

The authors declare that they have no conflict of interest.

\section{References}

1 Gesellschaft der epidemiologischen Krebsregister in Deutschland e.V.: Atlas der Krebsinzidenz und -mortalität in Deutschland (GEKID-Atlas). Lübeck, GEKID, 2017. www.gekid.de

2 Schmitz KH, Courneya KS, Matthews C, et al; American College of Sports Medicine: American College of Sports Medicine roundtable on exercise guidelines for cancer survivors. Med Sci Sports Exerc 2010;42:14091426.

3 Huy C, Schmidt ME, Vrieling A, Chang-Claude J, Steindorf K: Physical activity in a German breast cancer patient cohort: one-year trends and characteristics associated with change in activity level. Eur J Cancer 2012;48:297-304.
4 Lucas AR, Levine BJ, Avis NE: Posttreatment trajectories of physical activity in breast cancer survivors. Cancer 2017;123:2773-2780.

5 Friedenreich CM: The role of exercise in cancer progression and mortality: observational and molecular epidemiologic evidence. Cancer Res 2016;76:abstr SY22-01.

6 Buffart LM, Galvao DA, Brug J, Chinapaw MJ, Newton RU: Evidence-based physical activity guidelines for cancer survivors: current guidelines, knowledge gaps and future research directions. Cancer Treat Rev 2014; 40:327-340.

7 Zopf EM, Baumann FT, Pfeifer K: Körperliche Aktivität und körperliches Training in der Rehabilitation einer Krebserkrankung. Rehabilitation 2014;53:2-7.
8 Adraskela K, Veisaki E, Koutsilieris M, Philippou A: Physical exercise positively influences breast cancer evolution. Clin Breast Cancer 2017;17:408-417.

9 Volaklis KA, Halle M, Tokmakidis SP: Exercise in the prevention and rehabilitation of breast cancer. Wien Klin Wochenschr 2013;125:297-301.

10 Runowicz CD, Leach CR, Henry NL, et al: American Cancer Society/American Society of Clinical Oncology Breast Cancer Survivorship Care Guideline. J Clin Oncol 2016;34:611-635.

11 Neil-Sztramko SE, Winters-Stone KM, Bland KA, Campbell KL: Updated systematic review of exercise studies in breast cancer survivors: attention to the principles of exercise training. Br J Sports Med 2017;DOI: 10.1136/bjsports-2017-098389. 
12 Solheim TS, Laird BJA, Balstad TR, et al: Cancer cachexia: rationale for the MENAC (Multimodal-Exercise, Nutrition and Anti-inflammatory medication for Cachexia) trial. BMJ Support Palliat Care 2018;DOI: 10.1136/bmjspcare-2017-001440.

13 Bluethmann SM, Vernon SW, Gabriel KP, Murphy CC, Bartholomew LK: Taking the next step: a systematic review and meta-analysis of physical activity and behavior change interventions in recent post-treatment breast cancer survivors. Breast Cancer Res Treat 2015;149:331-342.

14 Spence RR, Heesch KC, Brown WJ: Exercise and cancer rehabilitation: a systematic review. Cancer Treat Rev 2010;36:185-194.

15 Cox M, Basen-Engquist K, Carmack CL, et al: Comparison of internet and telephone interventions for weight loss among cancer survivors: randomized controlled trial and feasibility study. JMIR Cancer 2017;3: e16.

16 Ritvo P, Obadia M, Santa Mina D, et al: Smartphoneenabled health coaching intervention (iMOVE) to promote long-term maintenance of physical activity in breast cancer survivors: protocol for a feasibility pilot randomized controlled trial. JMIR Res Protoc 2017; 6:e165.

17 Swartz MC, Lewis ZH, Lyons EJ, et al: Effect of homeand community-based physical activity interventions on physical function among cancer survivors: a systematic review and meta-analysis. Arch Phys Med Rehabil 2017;98:1652-1665.

18 Kirkham AA, Van Patten CL, Gelmon KA, et al: Effectiveness of oncologist-referred exercise and healthy eating programming as a part of supportive adjuvant care for early breast cancer. Oncologist 2018;23:105115.

19 Schmitt J, Lindner N, Reuss-Borst M, Holmberg HC, Sperlich B: A 3-week multimodal intervention involving high-intensity interval training in female cancer survivors: a randomized controlled trial. Physiol Rep 2016;4:e12693.

20 Schulz SVW, Laszlo R, Otto S, et al: Feasibility and effects of a combined adjuvant high-intensity interval/ strength training in breast cancer patients: a singlecenter pilot study. Disabil Rehabil 2018;40:1501-1508.

21 Mazor M, Lee JQ, Peled A, et al: The effect of yoga on arm volume, strength, and range of motion in women at risk for breast cancer-related lymphedema. J Altern Complement Med 2018;24:154-160.

22 Melchiorri G, Viero V, Triossi T, et al: New approach to evaluate late arm impairment and effects of dragon boat activity in breast cancer survivors. Medicine (Baltimore) 2017;96:e8400.

23 De Jesus S, Fitzgeorge L, Unsworth K, et al: Feasibility of an exercise intervention for fatigued breast cancer patients at a community-based cardiac rehabilitation program. Cancer Manag Res 2017;9:29-39.

24 Dittus KL, Lakoski SG, Savage PD, et al: Exercise-based oncology rehabilitation: leveraging the cardiac rehabilitation model. J Cardiopulm Rehabil Prev 2015;35: 130-139.

25 Penttinen HM, Saarto T, Kellokumpu-Lehtinen P, et al: Quality of life and physical performance and activity of breast cancer patients after adjuvant treatments. Psychooncology 2011;20:1211-1220.

26 Mustian KM, Sprod LK, Palesh OG, et al: Exercise for the management of side effects and quality of life among cancer survivors. Curr Sports Med Rep 2009;8: 325-330.

27 Duncan M, Moschopoulou E, Herrington E, et al; SURECAN Investigators: Review of systematic reviews of non-pharmacological interventions to improve quality of life in cancer survivors. BMJ Open 2017; 7:e015860.
28 Mirandola D, Miccinesi G, Muraca MG, Sgambati E, Monaci M, Marini M: Evidence for adapted physical activity as an effective intervention for upper limb mobility and quality of life in breast cancer survivors. J Phys Act Health 2014;11:814-822.

29 Mustian KM, Alfano CM, Heckler C, et al: Comparison of pharmaceutical, psychological, and exercise treatments for cancer-related fatigue: a meta-analysis. JAMA Oncol 2017;3:961-968.

30 Zimmer P, Baumann FT, Oberste M, et al: Influence of personalized exercise recommendations during rehabilitation on the sustainability of objectively measured physical activity levels, fatigue, and fatigue-related biomarkers in patients with breast cancer. Integr Cancer Ther 2017;DOI: 10.1177/1534735417713301.

31 Meneses-Echavez JF, Gonzalez-Jimenez E, Correa JE, Ramirez-Velez R: Supervised physical activity interventions in the management of cancer-related fatigue: a systematic review (Article in Spanish). Nutr Hosp 2014;30:486-497.

32 McNeely ML, Courneya KS: Exercise programs for cancer-related fatigue: evidence and clinical guidelines. J Natl Compr Canc Netw 2010;8:945-953.

33 Juvet LK, Thune I, Elvsaas IKO, et al: The effect of exercise on fatigue and physical functioning in breast cancer patients during and after treatment and at 6 months follow-up: a meta-analysis. Breast 2017;33: 166-177.

34 Streckmann F, Zopf EM, Lehmann HC, et al: Exercise intervention studies in patients with peripheral neuropathy: a systematic review. Sports Med 2014;44: 1289-1304.

35 Kleckner IR, Kamen C, Gewandter JS, et al: Effects of exercise during chemotherapy on chemotherapy-induced peripheral neuropathy: a multicenter, randomized controlled trial. Support Care Cancer 2018;26: 1019-1028.

36 Zimmer P, Baumann FT, Oberste M, et al: Effects of exercise interventions and physical activity behavior on cancer related cognitive impairments: a systematic review. Biomed Res Int 2016;2016:1820954.

37 Hartman SJ, Nelson SH, Myers E, et al: Randomized controlled trial of increasing physical activity on objectively measured and self-reported cognitive functioning among breast cancer survivors: the Memory\&Motion study. Cancer Cytopathol 2018;124:192-202.

38 Baumann FT, Reike A, Reimer V, et al: Effects of physical exercise on breast cancer-related secondary lymphedema: a systematic review. Breast Cancer Res Treat 2018;DOI: 10.1007/s10549-018-4725-y.

39 Rogan S, Taeymans J, Luginbuehl H, Aebi M, Mahnig S, Gebruers N: Therapy modalities to reduce lymphoedema in female breast cancer patients: a systematic review and meta-analysis. Breast Cancer Res Treat 2016; 159:1-14.

40 Letellier ME, Towers A, Shimony A, Tidhar D: Breast cancer-related lymphedema: a randomized controlled pilot and feasibility study. Am J Phys Med Rehabil 2014;93:751-759; quiz 760-761.

41 Irwin ML, Cartmel B, Gross CP, et al: Randomized exercise trial of aromatase inhibitor-induced arthralgia in breast cancer survivors. J Clin Oncol 2015;33:11041111.

42 Yang GS, Kim HJ, Griffith KA, Zhu S, Dorsey SG, Renn CL: Interventions for the treatment of aromatase inhibitor-associated arthralgia in breast cancer survivors: a systematic review and meta-analysis. Cancer Nurs 2017;40:E26-E41.

43 Jones LW, Habel LA, Weltzien E, et al: Exercise and risk of cardiovascular events in women with nonmetastatic breast cancer. J Clin Oncol 2016;34:2743-2749.

44 Emaus A, Veierod MB, Tretli S, et al: Metabolic profile, physical activity, and mortality in breast cancer patients. Breast Cancer Res Treat 2010;121:651-660.
45 Bertram LA, Stefanick ML, Saquib N, et al: Physical activity, additional breast cancer events, and mortality among early-stage breast cancer survivors: findings from the WHEL Study. Cancer Causes Control 2011; 22:427-435

46 Irwin ML, Smith AW, McTiernan A, et al: Influence of pre- and postdiagnosis physical activity on mortality in breast cancer survivors: the health, eating, activity, and lifestyle study. J Clin Oncol 2008;26:3958-3964.

47 Adamo R, Klika RJ, Ballard TM: How does physical activity prior to breast cancer diagnosis effect rehabilitation outcomes? Southern California, SCCUR FALL Conference, 2016.www.sccur.org/sccur/fall_2016_conference/posters/220/.

48 Jakobisiak M, Lasek W, Golab J: Natural mechanisms protecting against cancer. Immunol Lett 2003;90:103122.

49 Hutnick NA, Williams NI, Kraemer WJ, et al: Exercise and lymphocyte activation following chemotherapy for breast cancer. Med Sci Sports Exerc 2005;37:1827-1835.

50 Do J, Cho Y, Jeon J: Effects of a 4-week multimodal rehabilitation program on quality of life, cardiopulmonary function, and fatigue in breast cancer patients. J Breast Cancer 2015;18:87-96.

51 Loprinzi PD, Cardinal BJ, Winters-Stone K, Smit E Loprinzi CL: Physical activity and the risk of breast cancer recurrence: a literature review. Oncol Nurs Forum 2012;39:269-274.

52 Brunet J, St-Aubin A: Fostering positive experiences of group-based exercise classes after breast cancer: what do women have to say? Disabil Rehabil 2016;38:1500-1508.

53 Baumann FT, Bieck O, Oberste M, et al: Sustainable impact of an individualized exercise program on physical activity level and fatigue syndrome on breast cancer patients in two German rehabilitation centers. Support Care Cancer 2017;25:1047-1054.

54 Friedenreich CM, Neilson HK, Farris MS, Courneya KS Physical activity and cancer outcomes: a precision medicine approach. Clin Cancer Res 2016;22:4766-4775.

55 Basen-Engquist K, Alfano CM, Maitin-Shepard M, et al: Agenda for translating physical activity, nutrition, and weight management interventions for cancer survivors into clinical and community practice. Obesity (Silver Spring) 2017;25(suppl 2):S9-S22.

56 Stubblefield MD: The underutilization of rehabilitation to treat physical impairments in breast cancer survivors. PM R 2017;9:S317-S323.

57 Cheville AL, Mustian K, Winters-Stone K, Zucker DS, Gamble GL, Alfano CM: Cancer rehabilitation: an overview of current need, delivery models, and levels of care. Phys Med Rehabil Clin N Am 2017;28:1-17.

58 Van Waart H, van Dongen JM, van Harten WH, et al: Cost-utility and cost-effectiveness of physical exercise during adjuvant chemotherapy. Eur J Health Econ 2017;DOI: 10.1007/s10198-017-0936-0.

59 Pennington KP, McTiernan A: The role of physical activity in breast and gynecologic cancer survivorship. Gynecol Oncol 2018;DOI: 10.1016/j.ygyno.2018.01.020.

60 Leclerc AF, Foidart-Dessalle M, Tomasella M, et al Multidisciplinary rehabilitation program after breast cancer: benefits on physical function, anthropometry and quality of life. Eur J Phys Rehabil Med 2017;53: 633-642.

61 Dolan LB, Barry D, Petrella T, et al: The cardiac rehabilitation model improves fitness, quality of life, and depression in breast cancer survivors. J Cardiopulm Rehabil Prev 2017;DOI: 10.1097/HCR.0000000000000256.

62 Rock CL, Doyle C, Demark-Wahnefried W, et al: Nutrition and physical activity guidelines for cancer survivors. CA Cancer J Clin 2012;62:243-274.

63 Sisler J, Chaput G, Sussman J, Ozokwelu E: Follow-up after treatment for breast cancer: practical guide to survivorship care for family physicians. Can Fam Physician 2016;62:805-811. 
64 Ibrahim EM, Al-Homaidh A: Physical activity and survival after breast cancer diagnosis: meta-analysis of published studies. Med Oncol 2011;28:753-765.

65 Zhong S, Jiang T, Ma T, Zhang X, Tang J, Chen W, Lv $\mathrm{M}$, Zhao J: Association between physical activity and mortality in breast cancer: a meta-analysis of cohort studies. Eur J Epidemiol 2014;29:391-404.

66 Schmid D, Leitzmann MF: Association between physical activity and mortality among breast cancer and colorectal cancer survivors: a systematic review and meta-analysis. Ann Oncol 2014;25:1293-1311.
67 Nelson SH, Marinac CR, Patterson RE, et al: Impact of very low physical activity, BMI, and comorbidities on mortality among breast cancer survivors. Breast Cancer Res Treat 2016;155:551-557.

68 Schmidt ME, Chang-Claude J, Vrieling A, et al: Association of pre-diagnosis physical activity with recurrence and mortality among women with breast cancer. Int J Cancer 2013;133:1431-1440.

69 De Glas NA, Fontein DB, Bastiaannet E, et al: Physical activity and survival of postmenopausal, hormone receptor-positive breast cancer patients: results of the Tamoxifen Exemestane Adjuvant Multicenter Lifestyle study. Cancer 2014;120:2847-2854.
70 Lahart IM, Metsios GS, Nevill AM, Carmichael AR: Physical activity, risk of death and recurrence in breast cancer survivors: a systematic review and meta-analysis of epidemiological studies. Acta Oncol 2015;54: 635-654.

71 Holmes MD, Chen WY, Feskanich D, Kroenke CH, Colditz GA: Physical activity and survival after breast cancer diagnosis. JAMA 2005;293:2479-2486. 